\title{
Author Correction: Overanxious and underslept
}

\author{
Eti Ben Simon (1D, Aubrey Rossi, Allison G. Harvey and Matthew P. Walker
}

Correction to: Nature Human Behaviour https://doi.org/10.1038/s41562-019-0754-8, published online 4 November 2019.

In the version of this article initially published, several statistical values were not correct. These errors resulted from the fact that incorrect values from an earlier draft were inadvertently left in the final submission. Notably, the corrections do not change the significance (or lack thereof) of any of the results presented in the paper. As a consequence, the scientific conclusions drawn from all statistical analyses described in the manuscript remain unchanged.

These corrections comprise four numerical revisions in the in-laboratory study and two revisions in the online study. Specifically:

- In the section "In-laboratory study: sleep-loss-induced anxiety" the last sentence of the second paragraph should read: "SD [sleep deprivation] resulted in a $27 \%$ increase in anxiety relative to the SR [sleep rested] condition $(\mathrm{SR}=-0.7 \pm 3.31, \mathrm{SD}=27.12 \pm 7.35$, mean difference $=27.82 \pm 9.06,95 \% \mathrm{CI}=[8.7,46.93], t(17)=3.07, P=0.007)$."

- The legend for Fig. 1c should read, "Overnight changes in anxiety levels revealed a $27 \%$ increase in anxiety following SD, yet no change following the SR night (sleep $\times$ time interaction, $F(1,16)=8.95, \eta^{2}=0.34, P=0.008$ )."

- In the section "In-laboratory study: sleep physiology in relation to anxiety and emotional brain dynamics" the first sentence of the second paragraph should read: "Consistent with the prediction, NREM SWS predicted the degree of overnight reduction in anxiety from evening to morning (within-subject measure, $R=-0.49,95 \% \mathrm{CI}=[-0.02,-0.79], P=0.044$ ) in the SR condition and predicted the outright next-morning anxiety score $(R=-0.59,95 \% \mathrm{CI}=[-0.15,-0.8], P=0.01$; Fig. $4 \mathrm{a})$."

- The first sentence of the following paragraph should read: "Importantly, the association between NREM SWS and anxiety was specific to state anxiety, with no significant association with trait anxiety scores $\left(R=-0.25, P=0.3 ; \mathrm{BF}_{01}=2.12,95 \% \mathrm{CI}[0.26,-0.66]\right)$."

Again, all results that were significant remain significant, and all those that were non-significant remain non-significant. As such, all conclusions drawn from the in-laboratory study remain the same.

- In the section "Online micro-longitudinal studies 1 and 2: night-to-night perturbations in sleep quality negatively impact next-day anxiety" the first sentence of the second paragraph should read: "Supporting the experimental prediction, and the directional predictions from the in-laboratory study, there was a significant association between night-to-night changes in sleep efficiency and subsequent day-to-day changes in experienced anxiety: individuals who experienced a reduction in sleep efficiency from one night to the next reported a corresponding and significant increase in next-day anxiety, and vice versa $(F(1,93)=4.39$, mean change $=0.3 \pm 0.46$ and $-0.98 \pm 0.39$, respectively, $\left.\eta^{2}=0.053, P=0.039\right)$.”

- The last sentence of the fourth paragraph should read: "These analyses revealed that the association between sleep efficiency and anxiety is evident even when controlling for previous anxiety states (online study $1, R=-0.15, P=0.034$; online study 2 , $\beta=-16.94$, $t(48.37)=-4.83, P<0.0001)$."

Once again, all findings that were significant remain significant, and thus all conclusions drawn from the results of the online study remain unchanged. The errors have been corrected in the PDF and HTML versions of this article.

Published online: 30 November 2020

https://doi.org/10.1038/s41562-020-01016-1

(c) The Author(s), under exclusive licence to Springer Nature Limited 2020 